\title{
A Relação entre Variáveis de Saúde Mental e Cognição em Idosos Viúvos
}

\section{The Relation among Variables of Mental Health and Cognition in Widowed Elders}

\author{
Clarissa Marceli Trentini ${ }^{*}, a$, Blanca Susana Guevara Werlang ${ }^{b}$, \\ Flávio Merino de Freitas Xavier ${ }^{c} \&$ Irani Iracema de Lima Argimon ${ }^{b}$ \\ ${ }^{a}$ Universidade Federal do Rio Grande do Sul \\ ${ }^{b}$ Pontifícia Universidade Católica do Rio Grande do Sul \\ ${ }^{c}$ Universidade do Extremo Sul Catarinense
}

\begin{abstract}
Resumo
O objetivo do estudo foi avaliar as habilidades cognitivas de idosos viúvos. O grupo total de 34 viúvos (com perda do companheiro nos últimos 12 meses) foi identificado entre os idosos de Veranópolis (RS), sendo que 30 aceitaram participar do estudo. Outros 30 participantes casados, pareados conforme sexo, idade e escolaridade, compuseram o grupo controle. Os instrumentos utilizados foram: Questionário de Medida de Queixas Subjetivas de Memória (Mac-Q); Fluência verbal, categoria animal; Mini-exame do estado mental (MEEM); Teste de evocação seletiva livre e com pistas de Buschke; subteste de Dígitos da escala WAIS; Escala de depressão geriátrica (GDS) e Questionário fenomenológico do luto (BPQ). Verificou-se que os viúvos tinham significativamente mais sintomas depressivos e mais pontos na escala de luto. Entretanto, não foi evidenciada diferença significativa entre as médias no desempenho cognitivo de idosos viúvos ou idosos casados. Discute-se que a escolha da viuvez censitária (ter o estado civil viúvo) e não do luto emocional (autoreferido) como critério de seleção pode ter influído na ausência de associação entre viuvez e disfunção cognitiva, entre outros.

Palavras-chave: Idosos; Viúvos; Habilidades cognitivas; Avaliação neuropsicológica; Viuvez.
\end{abstract}

\begin{abstract}
To assess the cognitive abilities of widowed elders, a total sample of 34 elders (who have lost their spouses in the last 12 months) was identified among elders in Veranópolis-RS and 30 of them accepted to participate in the study. The control group was composed by 30 married subjects paired according to gender, age and level of education. The instruments used were: Measure Questionnaire of Memory Loss Complaints; Verbal Fluency - animal category; Mini-Mental State Examination (MMSE); Free and Cued Selective Reminding Test; Digit subtest; Geriatric Depression Scales and Bereavement Phenomenology Questionnaire. Widowed elders had significantly more depressive symptoms and more points on the bereavement scale. However, there was no significant difference between the means of cognitive performance of widowed or married elders. In the census, the choice of widowhood as a selection criterion instead of bereavement (self reported) might have influenced the lack of association between widowhood and cognitive dysfunction, among other aspects.

Keywords: Elders; Widows; Cognitive abilities; Neuropsychological assessment; Widowhood.
\end{abstract}

Existe um conjunto de evidências sugerindo que a depressão tem impacto na função cognitiva de pacientes idosos (Lamberty \& Bieliaukas, 1993; Raskin, Friedman, \& DiMascio, 1982), e recentes revisões sobre a assim denominada pseudodemência (termo que nomeia a perda cognitiva causada pela depressão entre idosos) estão disponíveis (Xavier, 2006). As dificuldades cognitivas do idoso com pseudodemência estão relacionadas tanto com a aten-

\footnotetext{
* Endereço para correspondência: Universidade Federal do Rio Grande do Sul, Instituto de Psicologia, Rua Ramiro Barcelos, 2600, sala 119, Porto Alegre, RS, Brasil, CEP 90035-003. Tel.: (51) 3308 5475. E-mail: clarissatrentini@terra.com.br

Agradecimentos: Conselho Nacional de Desenvolvimento Científico e Tecnológico (CNPq); Prefeitura Municipal de Veranópolis/RS.
}

ção (Cohen, Weingartner, Smallberg, Pickar, \& Murphy, 1993; Raskin et al., 1982), quanto com as habilidades visuoespaciais (Butters et al., 2000), com a velocidade psicomotora (Den Hartog, Derix, Van Bemmel, Kremer, \& Jolles, 2003; Nebes et al., 2000; Pálsson, Johansson, Berg, \& Skoog, 2000) e com a memória operacional de curta duração (Den Hartog et al., 2003; Lichtenberg, Ross, Millis, \& Manning, 1995; Nebes et al., 2000).

Ao mesmo tempo que a depressão está associada com disfunção cognitiva, também se sabe que o processo de luto normal na terceira idade está associado com um impacto negativo para diversos aspectos da saúde. Existem evidências associando o luto em idosos a um maior risco de transtorno mental (Bennett, 1998; Byrne, \& Raphael, 1994; GallagherThompson, Futterman, Farberow, Thompson, \& Peterson, 1993; Zisook, \& Schuchter, 1993), a um maior número de 
enfermidades de saúde geral (Helsing \& Szklo, 1981; Kaprio, Koskenvuo, \& Rita, 1987; Parkes, 1996), bem como a mortalidade aumentada (Bowling, 1987; Jones, 1987).

Segundo alguns autores (Chen et al., 1999; Prigerson et al., 1995; Prigerson et al., 1997; Xavier, Ferraz, Trentini, Freitas, \& Moriguchi, 2002) é provável que o impacto negativo do luto seja independente da variável depressão, muitas vezes sobreposta ao trabalho emocional da despedida. Em um estudo recente, Boelen e Prigerson (2007) demonstram que a associação do luto (pelo menos do luto mais intenso) com a qualidade de vida e com piores desfechos de saúde mental é independente da depressão e da ansiedade. Ott, Lueger, Kelber, e Prigerson (2007) identificaram também a associação específica do luto independente da depressão sobre a saúde geral e mental.

Assim, mesmo que existam evidências da associação de depressão com a disfunção cognitiva e de uma associação do luto com diversos desfechos clínicos (de qualidade de vida à mortalidade), pouco se investigou sobre uma possível associação de luto com disfunção cognitiva. Apenas cinco estudos com idosos nas últimas décadas em publicações em inglês tinham como finalidade o exame de uma possível associação entre luto e perda cognitiva, em analogia com a associação bem estabelecida entre depressão e perda cognitiva. Em um deles, Grimby (1993) observou que, entre 79 sujeitos idosos viúvos, um percentual de $40 \%$ relatava perda de memória e mais de $60 \%$ relatava problemas de concentração. O mesmo autor, em outro estudo (Grimby \& Berg, 1995), identificou que o único evento estressante de vida que tinha associação com a perda cognitiva era o luto. Também um estudo recente longitudinal (Aartsen, Van Tilburg, Smits, Comijs, \& Knipscheer, 2005) sugeriu que a perda do cônjuge pudesse estar associada com disfunção da memória em idosos. No mesmo sentido, em um estudo prévio na comunidade brasileira de Veranópolis, Xavier et al. (2002) encontraram pior desempenho cognitivo entre idosos enlutados do que entre controles. No sentido contrário, Ward, Mathias e Hitchings (2007) não conseguiram reproduzir estas evidências de pior padrão cognitivo de idosos enlutados em uma amostra na Austrália.

Em função de estar bem estabelecida tanto a associação entre depressão geriátrica e disfunção cognitiva, quanto depressão e luto (freqüentemente sobrepostos), todos os estudos acima precisaram controlar tanto a variável depressão quanto a variável ansiedade. São, portanto, com exceção do estudo de Ward et al. (2007), estudos que confirmam a impressão de independência da influência da variável depressão na disfunção cognitiva associada ao luto. Contudo, mesmo que progressivamente se entenda que luto e depressão são categorias diferentes e com independentes associações com os desfechos clínicos, na prática, a distinção clínica de ambas é, algumas vezes, complexa.

As dificuldades derivam dos seguintes aspectos: (a) alta comorbidade de depressão (seja com intensidade de DSM-IV - American Psychiatric Association [APA], 1998, ou subclínica) com o luto, estima-se que a prevalência de depressão maior no mês seguinte à perda seja de 33\% (Rosenzweig, Pasternak, Prigerson, Miller, \& Reynolds, 1996) e de depressão subclínica de $31 \%$ (Zisook, Paulus, Shuchter, \& Judd, 1997); (b) faz parte do luto usual a presença de alguns sintomas depressivos isolados como tristeza, insônia, insatisfação com o self, redução do apetite, perda de peso (Parkes, 2002; Zisook \& Shuchter, 1985); (c) um percentual menor de enlutados desenvolve um quadro de luto mais complicado, ou prolongado, sendo que entre esses sujeitos a intensidade de sintomas disfóricos é maior (Ott et al., 2007). Shear, Frank, Houck, e Reynolds (2005) apresentam uma comparação sobre como fazer o diagnóstico diferencial do luto complicado com o de depressão maior.

O foco do presente estudo é verificar se há associação de algum tipo de disfunção cognitiva com a perda recente do cônjuge em uma amostra de idosos da comunidade. É um estudo que procura expandir e confirmar o achado prévio na mesma comunidade de Veranópolis (Xavier et al., 2002), estudo esse que evidenciou presença de disfunção cognitiva entre enlutados. É um estudo com desenho e metodologia parecido com outro estudo realizado na Austrália (Ward et al., 2007) que, contrariando o esperado, não evidenciou para idosos uma associação entre viuvez e disfunção cognitiva. Neste sentido, como o estudo prévio brasileiro tem resultados divergentes do estudo australiano objetiva-se também, com o presente estudo, esclarecer a discrepância entre tais achados.

\section{Método}

\section{Participantes}

O poder público do município de Veranópolis, RS, em associação com o Instituto de Geriatria e Gerontologia da Pontifícia Universidade Católica do Rio Grande do Sul (PUCRS), desenvolvem e organizam pesquisas científicas com a população de sujeitos com mais de 60 anos da cidade, desde o ano de 1994. Com o objetivo de instrumentalizar as investigações gerontológicas, mantêm registro dos nomes e endereços dos idosos. Com base nesse banco de dados, foram contatados todos os 34 idosos viúvos, sujeitos com mais de 60 anos que perderam o cônjuge nos últimos 12 meses, sendo que 30 deles aceitaram participar da entrevista. Um segundo grupo, denominado de grupo controle, foi constituído de forma pareada para sexo, idade (faixas de anos, de 5 em 5 anos) e escolaridade com o grupo de viúvos, sendo que os participantes controles tinham estado civil casado. A escolha de sujeitos para o grupo controle foi por meio de sorteio randômico.

Para os dois grupos, foram excluídos idosos que tinham tido a ocorrência de luto por perdas de outras pessoas próximas, tais como filhos e netos - no último ano. Além disso, foram considerados os seguintes critérios como de exclusão: (a) ser solteiro/a; (b) já ter estabelecido outro relacionamento afetivo após a perda; (c) presença de demência; (d) história de AVC; (e) presença de delirium; e (f) não ser residente na comunidade. 


\section{Procedimentos}

Foi realizado um levantamento dos viúvos no município de Veranópolis, RS, e considerado o fator idade para a inclusão na amostra. As entrevistas foram realizadas no posto de saúde da cidade. Os/as idosos/as com dificuldade de locomoção, os residentes de áreas rurais ou os que assim desejassem, foram entrevistados em suas próprias casas. A partir do aceite através da assinatura do Termo de Consentimento Livre e Esclarecido, os idosos/as foram entrevistados individualmente pelos pesquisadores. $\mathrm{O}$ mesmo procedimento foi realizado com o grupo controle. Cada sujeito foi entrevistado apenas em uma ocasião, na qual os dados de identificação, a bateria neuropsicológica, a Escala de depressão geriátrica (GDS) para avaliar intensidade de depressão e o Questionário fenomenológico do luto (BPQ) para luto foram administrados, respeitando o critério indicado pelos juízes para estabelecimento da ordem dos instrumentos. A duração desse contato variou de $1 \mathrm{~h}$ a $1 \mathrm{~h} 30 \mathrm{~min}$.

\section{Instrumentos}

A partir de uma procura na literatura, foram escolhidos os instrumentos mais apropriados para a amostra em questão e elaborada uma seqüência para aplicação dos mesmos. Os instrumentos selecionados, conforme ordem de aplicação, para a investigação cognitiva foram:

Questionário de Medida de Queixas Subjetivas de Memória (Mac-Q) (Crook, Feher, \& Larrabee, 1992). Instrumento incluído com o objetivo maior de investigar a percepção subjetiva de memória. Apesar de amplamente usado em populações brasileiras (Argimon \& Stein, 2005; Mattos et al., 2003; Xavier, Ferraz, Gil, \& Moriguchi, 1999), este instrumento ainda não foi adequadamente traduzido e validado para o português.

Fluência Verbal - Categoria Animal (Spreen \& Beton, 1977). Trata-se de uma avaliação da capacidade de busca e recuperação de dados estabelecidos na memória de longa duração, exigindo habilidades de organização, autoregulação e memória operacional. Este teste é validado para o uso em nosso meio por Brucki (1996).

Mini-Exame do Estado Mental (MEEM) (Folstein, Folstein, \& McHugh, 1975). Um exame do estado mental abreviado, validado em nosso meio por Bertolucci, Brucki, Campacci e Juliano (1994).

Teste de Evocação Seletiva Livre e com Pistas (Buschke $\&$ Fuld, 1974). Avalia a memória na sua capacidade de armazenamento, retenção e recuperação (ou evocação) de memórias de longo-termo (Petersen, Smith, Ivnik, Kokmen, \& Tangalos, 1994; Petersen, Smith, Kokmen, Ivnik, \& Tangalos, 1992). Existem dois estudos brasileiros que disponibilizam médias e desvios deste instrumento para o nosso meio (Argimon \& Stein, 2005; Xavier et al., 2006).

Escala Wechsler de Inteligência para Adultos-Revisada ([WAIS-R]; Wechsler, 1981) - Subteste de Dígitos (categoria Verbal). Mede, basicamente, enquanto uma extensão da atenção, a retenção da memória imediata (dígitos na ordem direta), memória e capacidade de reversibilidade (dígitos na ordem inversa), concentração e tolerância ao estresse (Cunha, 2000).
Avaliações adicionais foram feitas com os seguintes instrumentos: Escala de Depressão Geriátrica (GDS) (Yesavage et al., 1983), que identifica e quantifica sintomas depressivos específicos para a população idosa (Sheikh \& Yesavage, 1986) validado em nosso meio por Paradela, Lourenço, e Veras (2005). A intensidade de sintomas de luto foram avaliadas fazendo uso de um instrumento ainda não validado para o uso em português, o Questionário fenomenológico do luto (BPQ) (Kissane, Bloch, \& McKenzie, 1997), que, para fins da atual pesquisa, foi traduzida e retrotraduzida por dois tradutores juramentados, seguindo os critérios recomendados para a utilização desse tipo de instrumento em nosso meio. $\mathrm{O}$ escore total foi adotado como medida para o estudo atual. A ordem de aplicação dos testes foi discutida e testada inicialmente em uma investigação piloto. Nesta ocasião, por meio do teste de Kappa, foi verificada a existência de concordância entre os aplicadores.

\section{Análise dos Dados}

As informações coletadas foram organizadas em um Banco de Dados no Programa Statistical Package for the Social Science 12.0. A análise exploratória dos dados foi realizada através de técnicas da estatística descritiva (média, desvio-padrão e distribuição de freqüências). Foi utilizado o teste- $t$ para amostras independentes e o coeficiente de correlação de Pearson ou Spearman, conforme distribuição, entre as variáveis. Também foi utilizada a análise de covariância. Para todas as análises, foi utilizado o nível de significância de $5 \%$.

\section{Considerações Éticas}

O projeto deste estudo foi submetido e aprovado pelo Comitê de Ética em Pesquisa da PUCRS.

\section{Resultados}

Devido ao pareamento, os dois grupos tiveram igual proporções de homens $(30 \%)$ e mulheres $(70 \%)$, igual faixa etária $(60-64$ anos $=6,7 \% ; 65-69$ anos $=30 \% ; 70-74$ anos $=$ $16,7 \% ; 75-79$ anos $=23,3 \% ; 80-84$ anos $=13,3 \% ; 85-89$ anos $=3,3 \% ; 90-94$ anos $=6,7 \%)$ e escolaridade similar $(0$ anos $=10 \% ; 1-5$ anos $=83,3 \% ; 9-11$ anos $=6,7 \%$ ).

A idade média do grupo dos viúvos foi de 74,10 anos $(D P=8,09)$, com idade mínima de 60 anos e máxima de 94 anos de idade. Para o grupo controle, a média de idade foi de 74,27 anos $(D P=8,03)$, com idade mínima de 60 anos e máxima de 90 anos de idade. Não houve diferença estatisticamente significativa entre os grupos para a variável idade $(p=0,936)$.

O grupo dos viúvos apresentou em média 3,40 anos de escolaridade formal $(D P=2,69)$, sendo a escolaridade mínima de 0 anos e a máxima de 11 anos. O grupo controle apresentou uma média de 3,70 anos de escolaridade formal $(D P=2,22)$, com uma escolaridade mínima de 0 anos e máxima de 9 anos. A comparação entre os grupos não mostrou diferença estatisticamente significativa para o número de anos de escolaridade $(p=0,639)$. 
Os dois grupos não diferiram de modo significativo quanto a predominância de local de nascimento (Veranópolis, RS), residência em Veranópolis (urbana), origem (Italiana), profissão (trabalhador especializado, funções manuais), situação ocupacional atual (aposentado inativo), atividades de lazer (sim), religião (católica), saúde referida (regular), limitação física (não), fumante (não) e quantidade de bebida alcoólica consumida (não consome).
Idosos viúvos e idosos controles não tinham um desempenho cognitivo significativamente diferente nos testes, conforme análise pelo teste $t$ para amostras independentes. Como representado na Tabela 1, o grupo de enlutados diferia dos controles quanto à intensidade de sintomas depressivos e quanto ao escore no Questionário Fenomenológico do Luto. Entre os casos, o número médio de sintomas depressivos e a média da intensidade do luto foram significativamente maiores do que os escores observados no grupo controle.

Tabela 1

Desempenho Cognitivo segundo os Diferentes Instrumentos Aplicados, em Ambos os Grupos (n=60)

\begin{tabular}{|c|c|c|c|c|c|c|}
\hline \multirow[b]{2}{*}{ Instrumentos } & \multicolumn{2}{|c|}{$\begin{array}{c}\text { Grupo } 1 \\
\text { Viúvos(as) recentes } \\
(n=30)\end{array}$} & \multicolumn{2}{|c|}{$\begin{array}{c}\text { Grupo } 2 \\
\text { Casados(as) } \\
(n=30)\end{array}$} & \multirow[t]{2}{*}{$t$} & \multirow[t]{2}{*}{$p$} \\
\hline & $M$ & $D P$ & $m$ & $D P$ & & \\
\hline Queixa subjetiva de memória (Mac-Q) & 25,73 & 4,20 & 27,33 & 4,51 & $-1,423$ & 0,160 \\
\hline Fluência verbal (FV) & 12,33 & 4,66 & 13,30 & 3,20 & $-0,938$ & 0,353 \\
\hline Minimental & 23,57 & 4,04 & 24,60 & 4,16 & $-0,976$ & 0,333 \\
\hline Buschke (Solp) & 93,47 & 5,65 & 94,77 & 3,10 & $-1,105$ & 0,274 \\
\hline Dígitos & 6,83 & 2,44 & 6,57 & 2,45 & 0,423 & 0,674 \\
\hline GDS & 5,13 & 3,44 & 3,37 & 3,31 & 2,028 & 0,047 \\
\hline Questionário Fenomenológico do Luto (BPQ) & 50,60 & 11,93 & 33,60 & 12,66 & 5,352 & $<0,001$ \\
\hline
\end{tabular}

Nota. Mac-Q= escore total do Questionário de medida de queixas subjetivas de memória; Fluência verbal= escore total do Teste de fluência verbal, categoria animal; Minimental= escore total do Mini-exame do estado mental; Solp= somatório de lembranças livres e com pistas, do Teste de Buschke; Dígitos= somatório bruto do subteste de Dígitos ordem direta e inversa, da Escala Wechsler de Inteligência (WAIS-R); GDS= escore total da Escala de depressão geriátrica; $\mathrm{BPQ}=$ Questionário Fenomenológico do Luto.

Também foram realizadas correlações entre o tempo da perda e os escores das escalas cognitivas, além da intensidade do luto e de depressão. Como pode ser verificado na Tabela 2, a seguir, foram verificadas associações significativas entre a percepção subjetiva de perda de memória e a intensidade de luto e depressão e entre a intensidade de sintomas de luto e sintomas depressivos. Além desses, uma correlação negativa foi encontrada entre o desempenho no teste Fluência verbal e a intensidade de sintomas depressivos. Desta forma, não foram evidenciadas correlações significativas entre intensidade do luto e o desempenho nos testes, embora se possa observar que aqueles com perdas afetivas tendem a avaliar-se como tendo pior memória e os sujeitos com maior intensidade de luto tinham também a percepção subjetiva de estarem com mais perdas de memória.

Tabela 2

Correlações entre Desempenho Cognitivo, Tempo da Perda, Intensidade do Luto e Depressão no Grupo de Viúvos/as (n=30)

\begin{tabular}{lccc}
\hline & Tempo da perda ${ }^{1}$ & TBPQ & TGDS \\
\hline Queixa subjetiva de memória (Mac-Q) & $-0,043(0,823)$ & $0,448(0,013)^{*}$ & $0,468(0,009) * *$ \\
Fluência verbal (FV) & $0,213(0,259)$ & $-0,106(0,579)$ & $-0,483(0,007) * *$ \\
Minimental & $-0,178(0,346)$ & $-0,203(0,281)$ & $-0,293(0,116)$ \\
Buschke (Solp) & $0,096(0,613)$ & $-0,166(0,382)$ & $-0,237(0,207)$ \\
Dígitos & $0,241(0,200)$ & $0,043(0,823)$ & $-0,326(0,078)$ \\
GDS & $0,070(0,714)$ & $0,425(0,019)^{*}$ & - \\
Questionário Fenomenológico do Luto & $-0,069(0,715)$ & $-0,069(0,715)$ & $0,070(0,714)$ \\
Tempo da perda & - & $-0,019) *$
\end{tabular}

Nota. ${ }^{1}$ Coeficiente de correlação de Spearman. ${ }^{*} p<0,05 . * * p<0,001$.

Um outro dado analisado foi o impacto da variável faixa etária sobre o desempenho cognitivo de viúvos mais e menos velhos e a variável escolaridade (medida em anos de estudo formal). Em relação a idade não foi evidenciada influência sobre o desempenho cognitivo, a escolaridade, entretanto, esteve significativamente correlacionada com pior desempenho de idosos viúvos: os enlutados com menor tempo de escolaridade tinham pior escores nos testes 
de Fluência Verbal, categoria animal ( $r=0,529 ; p=0,003)$, no Mini-Exame do Estado Mental $(r=0,484 ; p=0,007)$ e no subteste de Dígitos $(r=0,706 ; p<0,001)$. Apesar dessa correlação ser significativa ela não afetou a comparação do desempenho cognitivo em relação ao tempo de perda, sexo e intensidade do luto, esta conclusão foi obtida através da análise de covariância, controlando o efeito da escolaridade. Muito embora não fosse objetivo deste estudo, de modo complementar, vale citar que as mesmas correlações entre escolaridade e desempenho cognitivo foram encontradas entre os idosos casados (respectivamente $r=0,438$; $r=0,347$ e $r=0,331$, todos com $p<0,001)$.

\section{Discussão}

O presente estudo buscou resolver a discrepância de resultados entre dois estudos prévios, um na mesma comunidade - o estudo de Xavier et al. (2002) - e outro estudo australiano - de Ward et al. (2007). Enquanto o primeiro encontrou associação entre luto e disfunção cognitiva (de modo independente de depressão), o segundo evidenciou que a perda cognitiva presente não era independente da influência da depressão. Os resultados do presente estudo concordam com o estudo de Ward et al. e não confirmam os resultados de Xavier et al. (2002), na medida em que os enlutados desta amostra não tinham piores padrões cognitivos.

A metodologia empregada é muito semelhante a do estudo de Ward et al. (2007) e difere em aspectos importantes do estudo de Xavier et al. (2002), o que pode ser uma justificativa para concordar com os resultados do primeiro e diferir dos resultados do segundo. Se parece com o desenho do estudo de Ward et al. pela idade média dos participantes e pela definição de caso (sujeitos que perderam cônjuge).

Embora tenha sido realizado na mesma comunidade do estudo prévio de Xavier et al. (2002), o atual estudo possui importantes diferenças metodológicas com relação a esse. Estas diferenças poderiam justificar a não concordância dos achados. As principais diferenças com potencial de influir na discrepância de resultados seriam:

Idade Média dos Sujeitos. Xavier et al. (2002) estudaram uma amostra com idade média 10 anos mais do que a idade média dos sujeitos da presente amostra. No estudo deles, a idade média era em torno de 84 anos. Sujeitos de 74 anos diferem de sujeitos de 84 anos, e octogenários são classificados como idosos-idosos. Nesta faixa de velhice avançada, a fragilidade e a dependência são maiores, bem como o número de doenças crônicas (Lamberts, Beld, \& Lely, 1997) e o número de remédios em uso (Carvalho \& Garcia, 2003; Vaupel et al., 1998). Existe muita probabilidade de que o impacto de uma saúde física mais frágil, de um nível maior de dependência e mais fragilidade seja significativo sobre aspectos como depressão, cognição e reação emocional. Neste sentido, Onrust, Cuijpers, Smit e Bohlmeijer (2006) identificaram que a saúde física mais pobre (um padrão mais esperado entre idosos mais velhos), era fator de risco para maior intensidade de sintomatologia depressiva entre enlutados. Curiosamente, também este mesmo autor, identificou que eram os mais jovens, e não os mais velhos, que tinham pior adaptação emocional à perda.

Tempo entre o Falecimento do Cônjuge e a Entrevista. Na presente avaliação, como também no estudo de Ward et al. (2007), foram selecionados sujeitos com perda do cônjuge nos últimos 12 meses. Já no trabalho de Xavier et al. (2002), não havia limite de tempo entre a perda e a entrevista, bastando a família avaliar que o idoso "ainda estava sob o efeito emocional da perda". Thompson, GallagherThompson, Futterman, Gilewski e Peterson (1991). evidenciaram que no primeiro ano, tanto a intensidade da sintomatologia depressiva quanto a intensidade da reação de luto, são marcadas. Segundo os autores, contudo, no final do primeiro ano, enquanto a sintomatologia depressiva declina, a sintomatologia do luto se mantém presente até mais de 30 meses após a perda. Desta forma, é possível que sujeitos com menos e sujeitos com mais de 12 meses de luto tenham sintomatologias diferentes, principalmente com relação aos quadros de depressão sobreposta. Essa questão poderia explicar por que Ward et al. (2007) encontrou sintomatologia depressiva em enlutados enquanto, por outro lado, Xavier et al. (2002) não evidenciou.

Outra decorrência dessa diferença no tempo médio de luto entre os estudos seria a inclusão, no estudo de Xavier et al. (2002), de um número maior de idosos com lutos prolongados. Existem evidências que demonstram que reações de luto prolongado são mais freqüentemente propensas a serem um luto complicado (Boelen \& van den Bout, 2007). Este fato de Xavier et al. (2002) possivelmente ter selecionado reações de luto mais prolongadas poderia ter aumentado o número de lutos complicados naquela amostragem. Como definido por Boelen e Prigerson (2007), o luto complicado é um construto diferente do luto usual. Existiria, desta forma, a possibilidade de que na presente amostra (assim como na de Ward et al., 2007) existissem mais casos de luto normal (pelo pouco tempo médio entre a perda e a entrevista) e que, no estudo prévio em Veranópolis, existissem mais casos de luto prolongado ou complicado.

Definição Cartorial de Casos. Foram incluídos como "caso", seja no estudo de Ward et al. (2007) ou no presente estudo, os idosos com perda de conjugue nos últimos 12 meses. No estudo de Ward et al., controles não poderiam ter tido outros lutos nos últimos 12 meses e, no presente estudo, controles eram sujeitos casados, pareados para escolaridade e idade. Em ambos os estudos, a definição de caso foi baseada na informação do cartório do estado civil viúvo/a, sendo assim uma definição apenas "censitária". Já no estudo de Xavier et al. (2002), a definição de "enlutado" dependia de critérios emocionais: foram incluídos como enlutados sujeitos cuja família julgava "que estivessem ainda sob o efeito emocional de uma perda". Na definição de Xavier et al. (2002), ambos os critérios tinham que ser preenchidos, o sujeito tinha que se avaliar como estando "emocionalmente afetado pela perda" no momento da avaliação cognitiva, bem como a família precisava 
identificar "observável impacto no dia-a-dia da perda nas emoções ou comportamento da pessoa idosa".

Recentemente as duas definições mais conhecidas de luto complicado propostas por dois autores diferentes sofreram uma tentativa de fusão, com objetivo de resolver divergências de ambas e de propor um novo construto mais preciso que venha a ser incluído na futura edição da DSM-V (Boelen \& Prigerson, 2007). O conceito de luto complicado foi proposto de uma forma por Prigerson et al. (1999) e de outra forma diferente por Horowitz et al. (1997). A definição de Prigerson et al. (1999) não incluem a evitação como critério, e sugerem uma janela de duração de sintomas de 6 meses e não 14 meses como Horowitz et al. Além disso, Prigerson et al. (1999) incluem o impacto na vida diária (o distúrbio na função, por exemplo, social) como um ponto central, que Horowitz et al. (1997) não incluem. Em um estudo recente (Forstmeier \& Maercker, 2007) evidenciou-se que a prevalência de luto complicado segundo a primeira definição era de $0,9 \%$ e segundo a definição de Horowitz et al. (1997) subia para 4,2\%. Esse estudo mostrou ainda que a concordância entre ambos os construtos diferentes de luto complicado era baixa $(\mathrm{Kappa}=0,13) \mathrm{e}$ que raramente os sujeitos recebiam o mesmo diagnóstico de luto complicado simultaneamente por ambos os critérios. Com objetivo de melhorar a noção de luto disfuncional - aquele que diferiria da reação esperada não patológica à perda - uma força tarefa vem propondo um novo construto para ser incluído na DSM-V, provisoriamente denominado de "transtorno de luto prolongado". Este construto de "transtorno de luto prolongado" se parece com a proposta de "caso" de Xavier et al. (2002) no tocante aos seguintes aspectos: ( a) pede a presença de resposta emocional, cognitiva ou comportamental (são listados exemplos dessas respostas entre os critérios diagnósticos); (b) pede a presença de impacto significativo dessa emotividade sobre a funcionalidade.

No presente estudo, a definição de caso foi apenas censitária (presença no cartório de estado civil viúvo/a e não levou em conta tanto presença de resposta emocional quanto presença de impacto desta emotividade na funcionalidade. Desta forma, existiria uma menor probabilidade de que na presente amostra tenham sido selecionados muitos sujeitos seja com luto complicado seja com "transtorno do luto prolongado" do que a probabilidade deste tipo de sujeitos terem sido selecionados na amostra de Xavier et al. (2002).

Uma hipótese importante a ser testada seria a de que luto mais complicado, e não luto usual, é que estaria associado com disfunção cognitiva. Dados preliminares de Ward et al. (2007) sugerem este fato, ainda que o autor refira a impossibilidade de uma conclusão correta devido ao pequeno número de sujeitos de sua amostra com reação de luto mais severa. Fazendo um estudo de analise fatorial, Ott et al. (2007) encontraram 3 grupamentos de reação de luto entre 141 idosos que perderam o cônjuge: (a) cluster luto "comum", corresponderia a $49 \%$ da amostra. Sujeitos nesse grupo experimentam intensa sintomatologia depressiva e de luto logo depois da perda (no primeiro ano), mas esta sintomatologia declina ao longo do tempo; (b) cluster luto "resiliente". Um conjunto de $34 \%$ dos sujeitos apresenta uma reação com padrões de sofrimento bastante menor, seja da sintomatologia depressiva, seja na intensidade do luto. Os sujeitos nesse cluster se percebem mais preparados para a morte, e percebem a morte de forma mais pacífica que sujeitos nos outros clusters. Os escores de saúde mental e de qualidade de vida são maiores neste grupo; (c) cluster luto "crônico", no qual um conjunto de $17 \%$ dos viúvos se enquadraria. Tem como característica a presença dos mais altos escores de luto e depressão. Os sujeitos com essa reação enfrentam mais freqüentemente uma perda súbita e apresentam mais baixa auto-estima e tem um maior nível de dependência da pessoa perdida.

Esse estudo de Ott et al. (2007) destaca a diversidade de respostas emocionais à perda, sendo que existiria a possibilidade conceitual de que apenas idosos com reações mais disfuncionais é que estivessem em risco para a disfunção cognitiva.

Alem da ausência de associação entre viuvez e alteração na cognição, outros achados do presente estudo foram: (a) enlutados tiveram maior intensidade de depressão $(p=0,04)$; (b) a intensidade da depressão era proporcional a intensidade do luto; (c) a percepção subjetiva do idoso de disfunção cognitiva era tanto mais intensa quanto a maior a intensidade do luto e quanto maior a intensidade da sintomatologia depressiva. Essa associação de intensidade de sintomas depressivos com intensidade de sintomas de luto também foi evidenciada por outros autores (Blay, 2000; Bromberg, Kovács, Carvalho, \& Carvalho, 1996; Parkes, 1998). Concordando com o achado de maior número de queixas subjetivas de memória entre enlutados, Grimby (1993) demonstrou que um grupo de 79 sujeitos idosos viúvos relatava mais perda de memória e mais problemas de concentração.

O estudo foi incapaz de reproduzir evidências prévias de Xavier et al. (2002) de disfunção cognitiva entre enlutados. $\mathrm{O}$ fato dos enlutados deste trabalho serem definidos por critérios censitários e não emocionais, o fato da definição de caso presente não exigir alteração na funcionalidade, bem como a idade média menor dos sujeitos avaliados no presente estudo são referidos como possíveis correlatos desta discrepância de resultados. Uma outra diferença metodológica é o tempo de luto variável entre os dois estudos. Os fatos de provavelmente Xavier et al. (2002) ter um maior número de indivíduos com o recentemente definido "transtorno de luto prolongado", e de que naquele estudo foi evidenciada uma associação de disfunção cognitiva com o luto podem apontar para a hipótese de que apenas reações emocionais de luto mais intensas, incapacitantes e prolongadas é que estariam associadas com perdas cognitivas.

A possibilidade de se chegar a um entendimento melhor sobre se existe ou não associação de disfunção cognitiva com reações emocionais de luto dependerá, desta forma, de refinamentos metodológicos em estudos subseqüentes. Muito importante para o entendimento do assunto é o 
controle das seguintes variáveis: (a) A estrita aplicação do recém proposto critério de "transtorno de luto prolongado"; (b) a avaliação do QI pré-mórbido; (c) a avaliação prévia antes da viuvez do grau de dependência emocional entre os cônjuges; (d) a aplicação dos critérios de DSM (APA, 1998) para depressão, distimia e ansiedade generalizada; (e) o uso de amostras não clínicas e sim comunitárias; (f) o uso (após adequada validação para o português) de instrumentos de estresse e de luto. É provável que estes mesmos aspectos metodológicos expliquem as discrepâncias de resultados entre o presente estudo e os estudos prévios.

\section{Referências}

Aartsen, M. J., Van Tilburg, T., Smits, C. H., Comijs, H. C., \& Knipscheer, K. C. (2005). Does widowhood affect memory performance of older persons? Psychological Medicine, 35, 217-226.

American Psychiatric Association. (1998). DSM-IV: Manual diagnóstico e estatístico de transtornos mentais (4th ed.). Porto Alegre, RS: ArtMed.

Argimon, I. I. L., \& Stein, L. M. (2005). Habilidades cognitivas em indivíduos muito idosos: Um estudo longitudinal. Cadernos de Saúde Pública, 21, 64-72.

Bennett, K. M. (1998). Longitudinal changes in mental and physical health among elderly, recently widowed men. Mortality, 3, 265-273.

Bertolucci, P. H. F., Brucki, S. M. D., Campacci, S. R., \& Juliano, Y. (1994). O mini-exame do estado mental em uma população geral: Impacto da escolaridade. Arquivos de Neuropsiquiatria, $52,1-7$.

Blay, S. L. (2000). Fatores de risco psicossociais da depressão em idosos. In O. V. Forlenza, \& P. Caramelli (Eds.), Neuropsiquiatria geriátrica (pp. 309-313). São Paulo, SP: Atheneu.

Boelen, P. A., \& van den Bout, J. (2007). Complicated grief and uncomplicated grief are distinguishable constructs. Psychiatry Research, 157, 311-314. Retrieved October 26, 2007, from http://www.ncbi.nlm.nih.gov/sites/entrez?db=pubmed\&uid= $17916387 \& \mathrm{cmd}=$ showdetailview $\&$ indexed $=$ google

Boelen, P. A., \& Prigerson, H. G. (2007). The influence of symptoms of prolonged grief disorder, depression, and anxiety on quality of life among bereaved adults: A prospective study [Eletronic version]. European Archives of Psychiatry and Clinical Neurosciences, 257(8), 444-452. Retrieved October 26, 2007, from http://www.springerlink.com/content/ u4626h712515wp84/

Bowling, A. (1987). Mortality after bereavement: A review of the literature on survival periods and factors affecting survival. Social Science \& Medicine, 24, 117-124.

Bromberg, M. H. P. F., Kovács, M. J., Carvalho, M. M. M. J., \& Carvalho, V. A. (1996). Vida e morte: Laços da existência. São Paulo, SP: Casa do Psicólogo.

Brucki, S. M. D. (1996). Dados normativos para o uso do teste fluência verbal (categoria animal), em nosso meio. Tese de Doutorado não-publicada, Escola Paulista de Medicina, Universidade Federal de São Paulo, SP.

Buschke, H., \& Fuld, P. A. (1974). Evaluation of storage, retention, and retriving in disordered memory and learning. Neurology, 11, 1019-1025.

Butters, M. A, Becker, J. T., Nebes, R. D., Zmuda, M. D., Mulsant, B. H., Pollock, B. G., et al. (2000). Changes in cognitive functioning following treatment of late-life depression. The American Journal of Psychiatry, 157, 1949-1954.
Byrne, G., \& Raphael, B. (1994). A longitudinal study of bereavement phenomena in recently widowed elderly men. Psychological Medicine, 24, 411-421.

Carvalho, J. A. M., \& Garcia, R. A. (2003). The aging process in the brazilian population: A demographic approach. Cadernos de Saúde Pública, 19, 725-733.

Chen, J. H., Bierhals, A. J., Prigerson, H. G., Kasl, S. V., Mazure, C. M., \& Jacobs, S. (1999). Gender differences in the effects of bereavement-related psychological distress in health outcomes. Psychological Medicine, 29, 367-380.

Cohen, R. M., Weingartner, H., Smallberg, S. A., Pickar, D., \& Murphy, D. L. (1993). Effort and cognition in depression. Archives of General Psychiatry, 39, 593-598.

Crook, T. H., Feher, E. P., \& Larrabee, G. J. (1992). Assesment of memory complaints in age associated memory impairment: The Mac-Q. International Psychogeriatrics, 4, 165-176.

Cunha, J. A. (2000). Psicodiagnóstico (Vol. 5, 5. ed.). Porto Alegre, RS: ArtMed.

Den Hartog, H. M., Derix, M. M., Van Bemmel, A. L., Kremer, B., \& Jolles, J. (2003). Cognitive functioning in young and middle-aged unmedicated out-patients with major depression: Testing the effort and cognitive speed hypotheses. Psychological Medicine, 33, 1443-1451.

Folstein, M. F., Folstein, S. E., \& Mchugh, P. R. (1975). Minimental state: A practical method for grading the cognitive state of patients for the clinician. Journal of Psychiatric Research, 12(3), 189-198.

Forstmeier, S., \& Maercker A. (2007). Comparison of two diagnostic systems for Complicated Grief. Journal of Affective Disorders, 99, 203-211.

Gallagher-Thompson, D., Futterman, A., Farberow, N., Thompson, L. W., \& Peterson, J. (1993). The impact of spousal bereavement on older widows and widowers. In M. S. Stroebe, W. Stroebe, \& R. O. Hansson (Eds.), Handbook of bereavement: Theory, research, and intervention (pp. 227-239). Cambridge, MA: Cambridge University Press.

Grimby, A. (1993). Bereavement among elderly people: Grief reactions, post-bereavement hallucinations and quality of life. Acta Psychiatrica Scandinavica, 87, 72-80.

Grimby, A., \& Berg, S. (1995). Stressful life events and cognitive functioning in late life. Aging (Milano), 7, 35-39.

Helsing, K. J., \& Szklo, M. (1981). Mortality after bereavement. American Journal of Epidemiology, 114, 41-52.

Horowitz, M. J., Siegel, B., Holen, A., Bonanno, G. A., Milbrath, C., \& Stinson, C. H. (1997). Diagnostic criteria for complicated grief disorder. American Journal of Psychiatry, 154, 904-910.

Jones, D. R. (1987). Heart disease mortality following widowhood: Some results of the OPCS longitudinal study. Journal of Psychosomatic Research, 31, 325-333.

Kaprio, J., Koskenvuo, M., \& Rita, H. (1987). Mortality after bereavement: Prospective study of 95647 widowed persons. American Journal of Public Health, 77, 283-287.

Kissane, D. W., Bloch, S., \& McKenzie, D. P. (1997). The bereavement phenomenology questionnaire: A single factor only. Journal of Psychiatry, 31, 370-374.

Lamberts, S. W. J., Beld, A. W., \& Lely, A. J. (1997). The endocrinology of aging. Science, 278, 419-424.

Lamberty, G. J., \& Bieliaukas, L. A. (1993). Distinguishing between depression and dementia in the elderly: A review of neuropsychological findings. Archives of Clinical Neuropsychology, 8, 149-170.

Lichtenberg, P. A., Ross, T., Millis, S. R., \& Manning, C. A. (1995). The relationship between depression and cognition in older adults: A cross-validation study. Journals of Gerontology Series B: Psychological Sciences and Social Sciences, 50, 25-32. 
Mattos, P., Lino, V., Rizo, L., Alfano, A., Araújo, C., \& Raggio, R. (2003). Memory complaints and test performance in healthy elderly persons. Arquivos de Neuropsiquiatria, 61, 920-924.

Nebes, R. D., Butters, M. A., Mulsant, B. H., Pollock, B. G., Zmuda, M. D., Houck, P. R., et al. (2000). Decreased working memory and processing seep mediate cognitive impairment in geriatric depression. Psychological Medicine, 30, 679-691.

Onrust, S., Cuijpers, P., Smit, F., \& Bohlmeijer, E. (2006). Predictors of psychological adjustment after bereavement. International Psychogeriatric, 19, 921-934.

Ott, C. H., Lueger, R. J., Kelber, S. T., \& Prigerson, H. G. (2007). Spousal bereavement in older adults: Common, resilient, and chronic grief with defining characteristics. The Journal of Nervous and Mental Disease, 195, 332-341.

Pálsson, S., Johansson, B., Berg, S., \& Skoog, I. (2000). A population study on the influence of depression on neuropsychological functioning in 85-year-olds. Acta Psychiatrica Scandinavica, 101, 185-193.

Paradela, E. M. P., Lourenço, R. A., \& Veras, R. P. (2005). Validação da escala de depressão geriátrica em um ambulatório geral. Revista de Saúde Pública, 39, 918-923.

Parkes, C. M. (1996). Bereavement. In T. Kendrick, A. Tylee, \& P. Freeling (Eds.), The prevention of mental illness in primary care (pp. 74-87). New York: Cambridge: University Press.

Parkes, C. M. (1998). Luto: Estudos sobre a perda na vida adulta. São Paulo, SP: Summus.

Parkes, C. M. (2002). Grief: Lessons from the past, visions for the future. Death Studies, 26, 367-385.

Petersen, R. C., Smith, G. E., Ivnik, R. J., Kokmen, E., \& Tangalos, E. G. (1994). Memory function in very early Alzheimer's disease. Neurology, 44, 867-872.

Petersen, R. C., Smith, G., Kokmen, E., Ivnik, R. J., \& Tangalos, E. G. (1992). Memory function in normal aging. Neurology, 42, 396-401.

Prigerson, H. G., Bierhals, A. J., Kasl, S. V., Reynolds, C. F., Shear, M. K., Day, N., et al. (1997). Traumatic grief as a risk factor for mental and physical morbidity. The American Journal of Psychiatry, 154, 616-623.

Prigerson, H. G., Frank, E., Kasl, S. V., Reynolds, C. F., Anderson, B., Zubenko, G. S., et al. (1995). Complicated grief and bereavement-related depression as distinct disorders: Preliminary empirical validation in elderly bereaved spouses. The American Journal of Psychiatry, 152, 22-30.

Prigerson, H. G., Shear, M. K., Jacobs, S. C., Reynolds, C. F., Maciejewski, P. K., Davidson, J. R., et al. (1999). Consensus criteria for traumatic grief: A preliminary empirical test. British Journal of Psychiatry, 174, 67-73.

Raskin, A., Friedman, A. S., \& DiMascio, A. (1982). Cognitive and performance deficits in depression. Psychopharmacology Bulletin, 18, 196-202.

Rosenzweig, A. S., Pasternak, R. E., Prigerson, H. G., Miller, M. D., \& Reynolds, C. F. (1996). Bereavement-related depression in the elderly. Is drug treatment justified? Drugs Aging, 8, 323-328.

Shear, K., Frank, E., Houck, P. R., \& Reynolds, C. F. (2005). Treatment of complicated grief: A randomized controlled trial. The Journal of American Medical Association, 293, 2601-2608.

Sheikh, J. I., \& Yesavage, J. (1986). A geriatric depression scale (GDS): Recent evidence and development of a shorter version. Reviews in Clinical Gerontology, 5, 165-173.

Spreen, O., \& Benton, A. L. (1977). Neurosensory Center Comprehensive Examination for Aphasia (NCCEA). Victoria, TX: University of Victoria.
Thompson, L. W., Gallagher-Thompson, D., Futterman, A., Gilewski, M. J., \& Peterson, J. (1991). The effects of late-life spousal bereavement over a 30-month interval. Psychological Aging, 6, 434-441.

Vaupel, J. W., Carey, J. R., Christensen, K., Johnson, T. E., Yashin, A. I., Holm, N. V., et al. (1998). Biodemografic trajectories of longevity. Science, 280, 855-860.

Ward, L., Mathias, J. L., \& Hitchings, S. E. (2007). Relationships between bereavement and cognitive functioning in older adults. Gerontology, 53, 124-134.

Wechsler, D. (1981). WAIS R - Wechsler Adult Intelligence ScaleRevised. Cleveland, $\mathrm{OH}$ : Psychological Corporation.

Xavier, F. M. F. (2006). O transtorno cognitivo da depressão geriátrica ou a pseudodemência depressiva do idoso. In M. A. M. P. Parente (Ed.), Cognição e envelhecimento (pp. 205-224). Porto Alegre, RS: ArtMed.

Xavier, F. M. F., Argimon, I., Zuppo, L. P., Lucchesi, L., Heluany, C. C. V., \& Trentini, C. M. (2006). O desempenho em testes neuropsicológicos de octagenários não-dementes e com baixa escolaridade em duas comunidades do sul do Brasil. Psico (Porto Alegre), 37(3), 221-231.

Xavier, F. M. F., Ferraz, M. P. T., Gil, O. F., \& Moriguchi, E. H. (1999). Queixa subjetiva de perda de memória no idoso: Uma comparação da percepção dos sujeitos com a percepção dos familiares em um estudo da comunidade. Revista Brasileira de Neurologia, 35, 137-141.

Xavier, F. M., Ferraz, M. P., Trentini, C. M., Freitas, N. K., \& Moriguchi, E. H. (2002). Bereavement-related cognitive impairment in an oldest-old community-dwelling Brazilian sample. Journal of Clinical and Experimental Neuropsychology, 24, 294-301.

Yesavage, J. A., Brink, T. L., Rose, T. L., Lum, O., Huang, V., Adey, M., et al. (1983). Development and validation of a geriatric depression screening scale: A preliminary report. Journal of Psychiatric Research, 17, 37-49.

Zisook, S., Paulus, M., Shuchter, S. R., \& Judd, L. L. (1997). The many faces of depression following spousal bereavement. Journal of Affective Disorders, 45, 85-95.

Zisook, S., \& Shuchter, S. R. (1985). Time course of spousal bereavement. General Hospital Psychiatry, 7, 95-100.

Zisook, S., \& Schuchter, S. (1993). Untraumatic bereavement. Journal of Clinical Psychiatry, 54, 365-372.
Recebido: 09/09/2005

$1^{a}$ revisão: 06/03/2006

$2^{a}$ revisão: 01/11/2007

$3^{a}$ revisão: 03/04/2008

Aceite final: $15 / 04 / 2008$ 